\title{
Epidemiology of Hepatocellular Carcinoma in India
}

\author{
Subrat K. Acharya \\ Department of Gastroenterology, All India Institute of Medical Sciences, New Delhi 110029, India
}

\begin{abstract}
Indian data on epidemiology of HCC is not available. Cancer is not a reportable disease in India and the cancer registries in India are mostly urban. National cancer registry program of the Indian Council of Medical Research (ICMR) has been recently expanded to include 21 population based and 6 hospital based cancer registries. The last published registry data by ICMR available in the cancer registry website (www.ncrpindia.org) was in 2008 which provides information on various cancers from 2006 to 2008. The other source of information was the report published by International Agency for Research on Cancer (WHO). According to these available data the age adjusted incidence rate of hepatocellular carcinoma (HCC) in India for men ranges from 0.7 to 7.5 and for women 0.2 to 2.2 per 100,000 population per year. The male:female ratio for HCC in India is 4:1. The age of presentation varies from $\mathbf{4 0}$ to 70 years. According to a study conducted by verbal autopsy in 1.1 million homes representing the whole country, the age standardized mortality rate for HCC in India for men is 6.8/ 100,000 and for women is 5.1/100,000. According to another study the incidence of HCC in cirrhotics in India is $1.6 \%$ per year. The unpublished data from various tertiary care centers suggest that the incidence of HCC is increasing in India. There is a need for a multi-centric HCC registry under the aegis of INASL. ( $\mathrm{J}$ CuIN ExP HePATOL 2014;4:S27-S33)
\end{abstract}

$\mathrm{H}$ epatocellular carcinoma (HCC) is the most frequent cause of all liver cancers and constitutes $90 \%$ of cancers of liver globally. ${ }^{1}$ Even though cancers of lungs, breast and large intestine have been identified as three most common cancers, the three leading causes of annual death due to cancers include lungs, stomach and liver. ${ }^{1}$ Approximately 7.5 Lakhs of new cases of HCC per year occurs globally which makes HCC as the 5th common cause of cancers effecting human. ${ }^{1}$ The mortality in HCC is very high; about 7 Lakhs death due to HCC occur annually and has been estimated to be 3rd common cause of death due to cancers effecting human. ${ }^{1}$

In India, information on HCC is inadequate. From 1988 till December 2012, over two and half decades only 68 publications have been listed in the PUBMED, all from tertiary care center, on select areas and most studies included small samples. The cancer registries in India probably do not provide accurate estimates of HCC prevalence due to its

Keywords: epidemiology, HCC, India

Received: 9.8.2013; Accepted: 20.5.2014; Available online 20.6.2014

Address for correspondence: Subrat K. Acharya, Professor, Department of Gastroenterology, All India Institute of Medical Sciences, New Delhi 110029, India. Tel.: +91 11 26594934; fax: +91 1126589130

E-mails: subratacharya2004@yahoo.com; subratacharya@hotmail.com

Abbreviations: AAIR: age adjusted incidence rate; ASMR: age standardized mortality rate; HBCR: hospital based cancer registry; HCC: hepatocellular carcinoma; IARC: international agency for cancer research; ICMR: Indian Council of Medical Research; PBCR: population based cancer registry http://dx.doi.org/10.1016/j.jceh.2014.05.013 predominant urban locations and the source of information on cancers are from cytology, oncology sites, and municipal registers of death. HCC predominantly is diagnosed and treated by Gastroenterologists/Hepatologist/ Transplant Surgeons/G.I. Surgeons. These patients treated by the later doctors are presumably are not being listed in the registries. Further, the diagnosis of hepatocellular cancer is achieved frequently by non-invasive imaging technique rather than by histology/cytological techniques as used in almost all other cancers. Therefore the collated informations from oncology/cytology/pathology departments may not be having the records of a considerable proportion of HCC cases. Lastly cancers are not reportable disease in India.

With these limitations, the specific issues which this review intend to do includes the following:

1. How common is HCC in India?

2. Status of reporting of HCC to cancer registries in India?

3. HCC burden in tertiary care centers?

4. Causes of mismatch of documented and likely actual burden.

5. Regional differences in HCC burden in India.

\section{HOW COMMON IS HEPATOCELLULAR CARCINOMA IN INDIA?}

The source of information to assess the frequency of occurrence of HCC in India includes autopsy data, cancer registries and population based surveillance data. 


\section{Autopsy Data}

The prevalences of HCC described in autopsy series from India are very old and were published between 1966 to 1982. ${ }^{2,3}$ The autopsy data has been published earlier by Dhir and Mohandas in 1998, ${ }^{3}$ which is depicted in Table 1.

The autopsy data reveals that $0.2-1.9 \%$ of autopsy cases had HCC with a higher prevalence of HCC in Southeastern states of India. However, these information have been replaced by cancer registry data in India.

\section{Cancer Registry Data}

National cancer registry program was established by Indian Council of Medical Research (ICMR) in 1981. Initially, three hospital-based cancer registries (HBCRs) and three population-based cancer registries (PBCRs) were established. By now, 21 PBCRs and 6 HBCRs have been established. The last published registry data by ICMR available in the cancer registry website (ncrpindia. org) was in 2008 which provides information on various cancers from 2006 to 2008 . The present write-up includes the information retrieved from this later report to provide the recent trends of HCC in India, which has been depicted in Table 2.

The summary interpretations of data depicted in ICMR cancer registry are as follows:

a) In 8 of the 23 PBCRs HCC was not included in the first 10 common cancers and in all these centers the age adjusted incidence rate (AAIR) of HCC/100,000 population was between 1 and 1.5. In the remaining PBCRs the AAIR ranged from 2.1 to 7.5 per 100,000 population.

The AAIR for HCC for men ranged from 0.9 to 7.5 and for women it ranged from 0.2 to 2.2 per 100,000 population. The highest AAIR was reported from Sikkim and Mizoram (Table 2). However at Barshi, HCC constituted 7.5\% of all cancer and was the most common cause of all cancers

Table 1 Autopsy Data on HCC in India.

\begin{tabular}{lrc}
\hline Place & Autopsies (No.) & HCC (\%) \\
\hline India & & \\
\hline - Mumbai & 6000 & 0.2 \\
\hline - Mumbai & 4000 & 0.2 \\
• Agra & 1234 & 0.7 \\
• Guntur & 629 & 1.1 \\
\hline • Andhra Pradesh & 2789 & 1.6 \\
• Chennai & 1218 & 1.9 \\
\hline Bangladesh & 5450 & 0.2 \\
\hline Denmark & 14881 & 0.3 \\
USA (Boston) & 14000 & 0.6 \\
Japan & 401,381 & 3.1 \\
\hline Hong Kong & 1480 & 6.8 \\
\hline
\end{tabular}

recorded in the cancer registry. Table 3 provides the AAIR of HCC in various cancer registries in India as depicted by WHO, ${ }^{5}$ which was updated in 2011 and provides similar data on frequency of HCC in India.

IARC-WHO data indicate that Africa and East Asia has the highest frequency of HCC globally with an AAIR of 14 36 per 100,000 people and account for approximately $80 \%$ of HCC globally. ${ }^{5,6}$

b) As per cancer registry data, ${ }^{4} 4-8 \%$ of the cancers were due to HCC in at least 13 of 21 PBCRs. At these centers males predominantly had been documented to have HCC with a M:F at 4:1 and the median age ranged from 40 to 70 and the frequency of liver cancers seems to be higher with increasing age.

c) To assess trend of frequency of digestive tract cancer Yeole et al analyzed PBCRs in five sites (Mumbai, Bangalore, Chennai, Delhi and Bhopal) over almost two decades (1980-2003). ${ }^{?}$

The annual percentage change in AAIR for Gut and liver cancer is depicted in Table 4.

The Table 4 indicates that there is a significant increase in liver cancer frequency in Mumbai, Chennai and Bangalore cancer registry over two decades.

Without visible control strategy for HCC, this trend of increase in frequency of occurrence of HCC is likely to continue. Indeed, in 1998 ICMR cancer registry from all sites documented 10,000 HCC cases. ${ }^{4}$ In 2002, IARC collated data and reported 13,630 HCC cases from India. ${ }^{5}$ In 2009, ICMR cancer registries have documented 25,000 cases of HCC. This would indicates that either HCC frequency is increasing or are being diagnosed more often.

IF approximately we presume that AAIR for HCC in India is about $3-5 / 100,000$ population annually, than in a year, approximately 30,000 to 50,000 HCC would be occurring in India.

\section{Population Based Surveys \\ Hepatocellular Carcinoma Related Mortality in General Population}

Dixit et $\mathrm{al}^{8}$ published an important population based survey to identify the cancer related mortality in India. ${ }^{8}$ The study was conducted by verbal autopsy study in 1.1 million homes representing the whole country. People who died between 2001 and 2003 were included in the study and the deaths were projected for year 2010. For both sexes and all ages 122,429 deaths occurred in 2001-2003 and 7137 of these were attributable to cancers. In 2010, more than 556,000 cancer deaths were estimated in people of all ages and $72 \%$ of these deaths were between 30 and 69 years. In 2010 at all ages, rates of cancer deaths about $59 / 100,000$ for men and about 52/100,000 for women. Among men, the first four causes of mortality included 
Table 2 Population Based Cancer Registry (PBCR), ICMR (ncrpindia.org). (Report on First 20 PBCR in India (2006-2008)-25 Geographical Area Population 3.81 Lakhs-156 Lakhs: Mizoram/Delhi, [1st Jan 2006-31st December 2008].

\begin{tabular}{|c|c|c|c|c|c|c|}
\hline PBCRs & Population & Total cancer & Liver cancer & CR & AAIR & Comment \\
\hline Bangalore & $6,892,642$ & 12624 & Not in 10 leading causes & 2.2 & 4.5 & \\
\hline Barshi & $4,855,549$ & 4308 & $7.5 \% *$ (1st common) & $3.5^{*}$ & $4.7^{*}$ & $\begin{array}{l}\text { Males } \\
\text { FX }\end{array}$ \\
\hline Bhopal & $1,812,045$ & 39757 & $X($ not in 10$)$ & - & - & \\
\hline Chennai & $4,695,780$ & 15258 & $x$ & 1.2 & 2.1 & \\
\hline Delhi & $16,501,678$ & 26333 & $1.07-2 \%$ & 1.5 & 2.6 & \\
\hline Mumbai & $13,494,514$ & 33230 & $4.9 \%$ (6th common) & 3.4 & 5.2 & $\mathrm{FX}$ \\
\hline Cachar & $1,626,339$ & 2539 & $2.1 \%$ (10th common) & 2.0 & 2.8 & \\
\hline Dibrugarh & $1,285,538$ & 2521 & $x$ & - & - & \\
\hline Kamrup & $1,117,804$ & 3256 & $x$ & - & - & \\
\hline Manipur & $2,436,500$ & 3969 & 4.4\% (6th common) & 2.3 & 3.1 & $F X$ \\
\hline Mizoram & $1,043,775$ & 3380 & $3.8 \%$ (5th common) & 4.3 & 6.4 & \\
\hline Sikkim & 648,806 & 1155 & $8 \%$ (4th common) & 4.7 & 7.5 & \\
\hline Ahmedabad & $6,313,384$ & 11844 & $x$ & 1 & 1.7 & \\
\hline Aurangabad & $1,119,142$ & 1888 & $\mathrm{x}$ & - & - & \\
\hline Kolkata & $4,677,682$ & 9038 & $\mathrm{X}$ & - & - & \\
\hline Kollam & $2,704,837$ & 9030 & 3.7\% (8th common) & 4.5 & 4.1 & $F X$ \\
\hline Nagpur & $2,322,117$ & 5422 & $\mathrm{X}$ & 1.4 & 2.2 & \\
\hline Pune & $4,676,184$ & 8594 & $4.5 \%$ (10th common) & 2.5 & 3.8 & $F X$ \\
\hline Trivandrum & $1,160,104$ & 5755 & $3.6 \%$ (9th common) & 4.6 & 4.5 & $\mathrm{FX}$ \\
\hline \multirow[t]{3}{*}{ Basis of diagnosis } & $61-94 \%$ & \multicolumn{5}{|c|}{ At various center by tissue diagnose } \\
\hline & $0.2-20 \%$ & \multicolumn{5}{|l|}{ Radiology } \\
\hline & $<10 \%$ & \multicolumn{5}{|l|}{ Other method } \\
\hline
\end{tabular}

NB: $4.8 \%$ of all cancers are liver cancer.

Male preponderance in all center.

8/20 PBCR-do not list liver cancer in the first 10 causes of cancer.

Median age-40 year to 70 years.

With increasing age-Numbers of liver cancer higher.

oral, stomach, lung and liver cancer. In 2010 approximately 14,000 deaths would have occurred due to liver cancer with an age standardized mortality rate (ASMR) of 6.8/100,000 population. In women liver cancer was the 8 th common

Table 3 AAIR of HCC in India (IARC-WHO). ${ }^{5}$

\begin{tabular}{|c|c|c|c|c|c|c|}
\hline & \multicolumn{3}{|c|}{ Male } & \multicolumn{3}{|c|}{ Female } \\
\hline & Cases & ASR & Cum & Cases & ASR & Cum \\
\hline Chennai & 269 & 3.0 & 0.37 & 83 & 0.9 & 0.10 \\
\hline New Delhi & 491 & 2.4 & 0.28 & 232 & 1.3 & 0.16 \\
\hline Karunagappally & 34 & 3.9 & 0.43 & 8 & 0.8 & 0.10 \\
\hline Mumbai & 895 & 4.5 & 0.56 & 433 & 2.2 & 0.25 \\
\hline Nagpur & 65 & 1.6 & 0.19 & 35 & 0.9 & 0.10 \\
\hline Poona & 209 & 3.4 & 0.41 & 88 & 1.4 & 0.15 \\
\hline Trivandrum & 78 & 2.8 & 0.32 & 30 & 1.0 & 0.12 \\
\hline
\end{tabular}

International Agency for Research on Cancer (IARC-WHO).

Age-standardized (per 100,000) and cumulative 0-74 incidence (\%) rates http://Ci5.iarc.fr.

cause of cancer related mortality accounting for 12,000 death in 2010 with an ASMR of 5.1/100,000 population.

Incidence of Hepatocellular Carcinoma in High Risk Individuals

The well known high risk patient to develop HCC includes cirrhosis of liver of any cause. ${ }^{1}$ About $80 \%$ of $\mathrm{HCC}$ has been

Table 4 Annual Percentage Change in Age Adjusted Incidence Rates for Gut and Liver Cancer.

\begin{tabular}{llllll}
\hline Registry & Esophagus & Stomach & Colon & Rectum & Liver \\
\hline Mumbai & $-2.91^{* *}$ & $-2.45^{* *}$ & $0.64^{\mathrm{NS}}$ & $0.17^{\mathrm{NS}}$ & $1.74 * *$ \\
Chennai & $1.12^{*}$ & $-0.98^{*}$ & $2.07^{* *}$ & $2.39 * *$ & $0.25 *$ \\
Bangalore & $0.13^{\mathrm{NS}}$ & $-0.74^{\mathrm{NS}}$ & $2.08^{*}$ & $1.69 *$ & $1.54 *$ \\
Delhi & $-1.36 *$ & $-1.57^{*}$ & $1.71^{*}$ & $-0.36^{\mathrm{NS}}$ & $1.32^{\mathrm{NS}}$ \\
Bhopal & $1.24^{\mathrm{NS}}$ & $-6.13^{*}$ & $2.79^{\mathrm{NS}}$ & $2.75^{\mathrm{NS}}$ & $2.10^{\mathrm{NS}}$ \\
\hline
\end{tabular}

NS—Not significant, $* p<0.05, * * p<0.01$. 
reported globally in cirrhotic livers. ${ }^{1}$ The frequency of HCC in a cirrhotic may vary depending upon underlying etiology of cirrhosis, such as HBV, HCV, alcohol and nonalcoholic fatty liver disease. ${ }^{9}$

Reports from tertiary care centers in India on HCC indicate that $70-97 \%$ of patients with HCC at the time of diagnosis had underlying cirrhosis of liver. ${ }^{10,11}$ Long term cohort follow-up studies from Europe and USA indicate that annual frequency of HCC in HBV-cirrhosis, HCVcirrhosis and alcohol induced cirrhosis have been $2.2 \%$, $3.8 \%$ and $1.7 \%$ respectively. ${ }^{12,13}$ The frequency of HCC occurrence is East Asian countries is further higher. ${ }^{14,15}$ These annual frequencies at HCC in HBV-cirrhosis may further vary as per their HBeAg status, HBV DNA load, age, sex and status of liver function. ${ }^{1,9,12-15}$

The ICMR cancer registries in India have documented low incidence of HCC on comparison to South East Asia, Japan and European countries, despite the fact that there are about 40-45 million HBV carrier, 10 million HCV infected individuals in India.

The discrepancy between higher prevalence of HCC risk factor and objective cancer registry data have been attributed to lower reporting by many. Further, prospective longitudinal study among cirrhotic cohorts, to document HCC incidence in India is scarce.

The only Indian study by Paul et al conducted a prospective observational study to assess the HCC incidence among patients with child's A and B cirrhotic without having any HCC at enrollment $(n=194)$ who were followed up for a median duration of 44 months. ${ }^{16}$ Each patient had ultrasonography and AFP at 6 month interval and TPCT annually. During a cumulative 563 person years followup nine cases of HCC (all males) were detected with an annual incidence rate of $1.6 \%$ (95\% CI-0.07-3) ${ }^{16}$ The authors concluded that incidence rate of HCC among Indian patient with cirrhosis is intermediate between high rates in Japan for East and European countries. ${ }^{16}$

In absence of estimates of accurate burden of cirrhosis in India, the absolute number of HCC occurring in India in a year is difficult to assess. Mohandas et al discussed this issue in an editorial published in the Indian Journal of Gastroenterology in 2007. ${ }^{9}$ This editorial reported that even if HCC in HCV and alcohol associated HCC are ignored, and assuming that 45 million $\mathrm{HBV}$ infected individuals with a $15 \%$ presumable $\mathrm{HBeAg}+\mathrm{ve}$ frequency and $0.01 \%$ annual HCC frequency, an over whelming 68,000 new HCC cases are likely to occur in India among HBV associated patients with chronic liver disease. However, the international agency for cancer research (IARC) had estimated 13,630 primary liver cancer in India (9153 males, 4477 females) in India in 2002. In contrast, the number of primary liver cancers from seven population based cancer registries included in the 'Cancer incidence in five continents' (Vol 9, 1998-2002) was 3023 during 179 million person years. ' Therefore at least there exists a fivefold difference between documented an estimated annual frequency of occurrence of HCC in India. The possible reasons of such discrepancy have been discussed later in the review.

\section{STATUS OF REPORTING HEPATOCELLULAR CARCINOMA TO CANCER REGISTRIES IN INDIA AND CAUSES OF MISMATCH OF DOCUMENTED AND LIKELY ACTUAL BURDEN OF HEPATOCELLULAR CARCINOMA IN INDIA}

As per the cancer registry website (ncrpindia.org) by now there are 23 population based cancer registries and 6 hospital cancer registries in India.

Each cancer registry has dedicated staff and electronic data collation capabilities. The information on site of cancers, death due to cancer and other etiological and demographic profile of each cancer are collated by visit of cancer registry staff to cytology, oncology, pathology department of the hospitals of the area designated for each cancer registries. The municipal death registers are also included as the source of information. These data are further supervised, cleaned and analyzed by supervisors and experts of each cancer registry site.

As mentioned earlier there exists a discrepancy between documented frequency of HCC in cancer registry and estimated likely frequency of HCC. The reason for such discrepancy may be due to many reasons. It is possible that actual burden of HCC in India may be lower. Mohandas et $\mathrm{al}^{9}$ had provided elegant explanation for documented lower incidence of HCC which are as follows.

\section{Over Estimation of Hepatitis B Virus Infection in Indians}

A systematic review of 54 Indian studies reported true prevalence of HBV infection in non-tribal and tribal populations to be $2.4 \%$ (95\% CI-2.2-2.7) and $15.9 \%$ (95\% CI11.4-20.4) respectively. ${ }^{17}$ Therefore the estimated HCC due HBV annually may be between 25 and 35000 HCC cases per year. Further, a large proportion of HCC cases may be HBeAg-ve with low HBV DNA load which is associated with lower frequency of HCC. ${ }^{1}$

\section{Average Life Span of Indians is Still 64 Years}

The population based incidence of HCC peaks at 75 years. The incidence rate of HCC in 100,000 males by age groups $40-44,50-54,60-64$ and $70-74$ years are $1.6,5.7,12.9$ and 22.6 respectively.

\section{In India Horizontal Transmission of Hepatitis B Virus is More Common}

Vertical transmission more frequently documented in genotype B and C HBV, particularly in South East Asian 


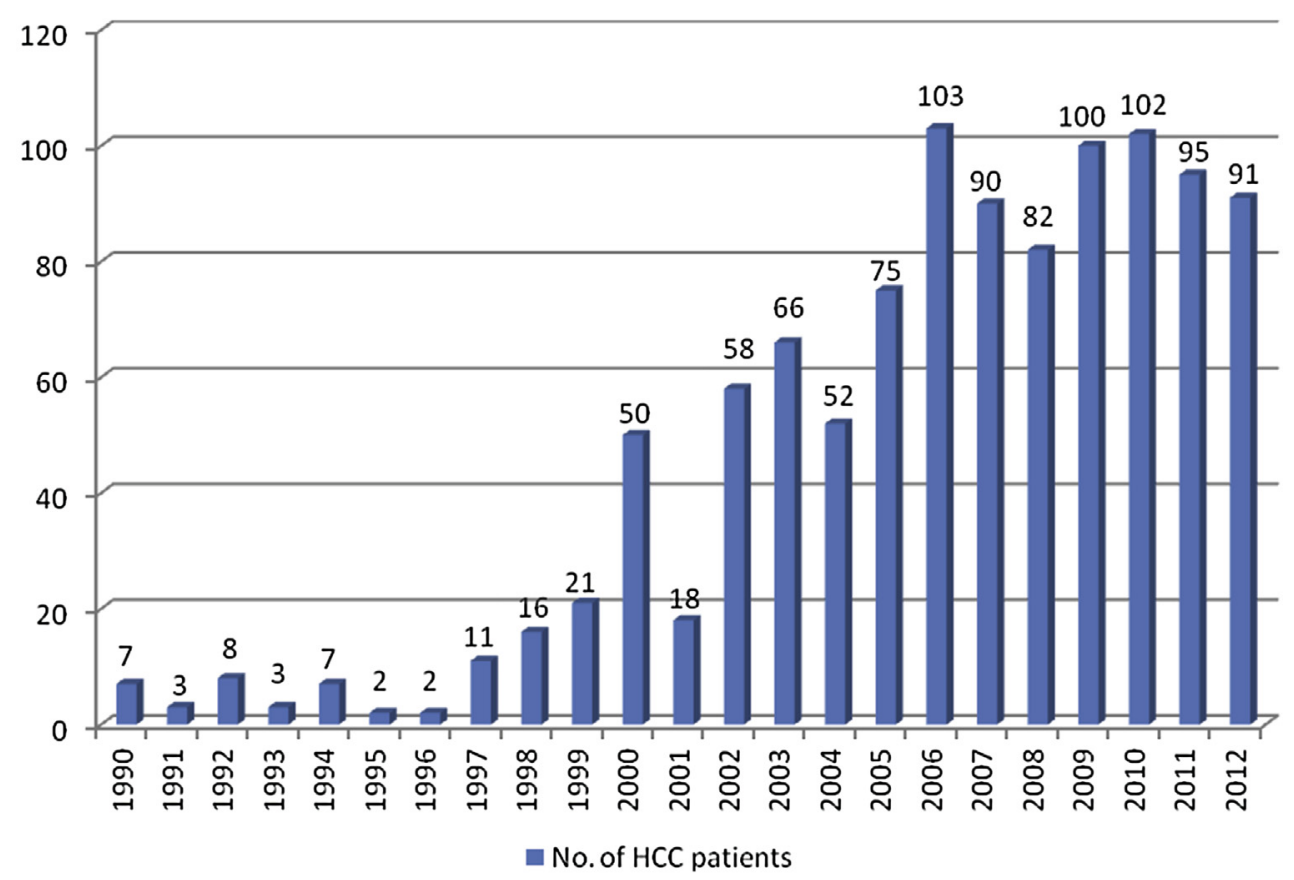

Figure 1 Year wise distribution of patients with HCC (AllMS 1990-2012).

countries which have been associated with high HCC incidence. In India, horizontal transmission of HBV infection with prevalence of HBV genotype A and D may be associated with lower incidence of HCC.

On the other hand it may be true that there is under reporting of HCC in the cancer registries. All the cancer registries in India indicate that most of the HCCs (61-95\%) documented had histological/cytological basis for the diagnosis of HCC. However, HCC during last decade is diagnosed mostly by imaging technique and FNAC is

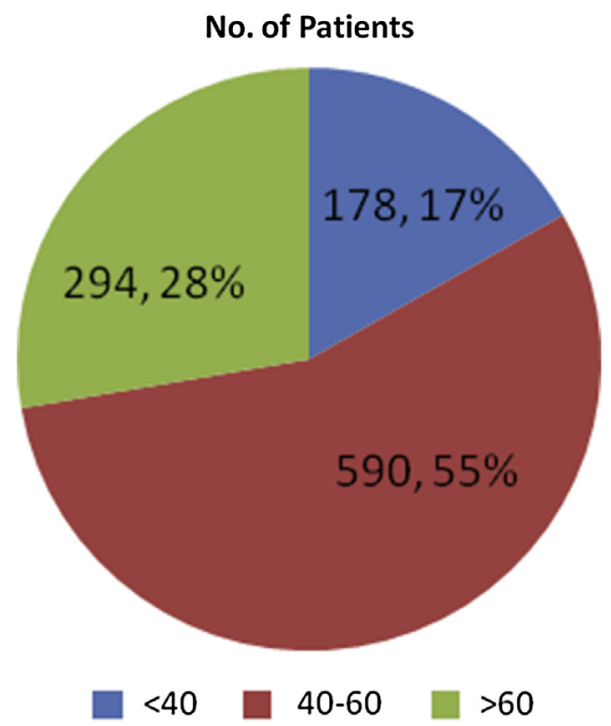

Figure 2 Age distribution of patients with HCC (AllMS 1990-2012). The figure indicates that the age distribution and $55 \%$ of these 1062 patients were above the age 62 years at the time of diagnosis. only performed in select number of cases in whom imaging characteristics of HCC are not evident. Further, most HCCs are managed by Gastroenterologists/Hepatologists and there is doubtful evidence that these patients are reported to the cancer registries.

\section{BURDEN OF HEPATOCELLULAR CARCINOMA IN TERTIARY CARE CENTER}

There are four large case series reports from India emanating from various tertiary care centers in India which

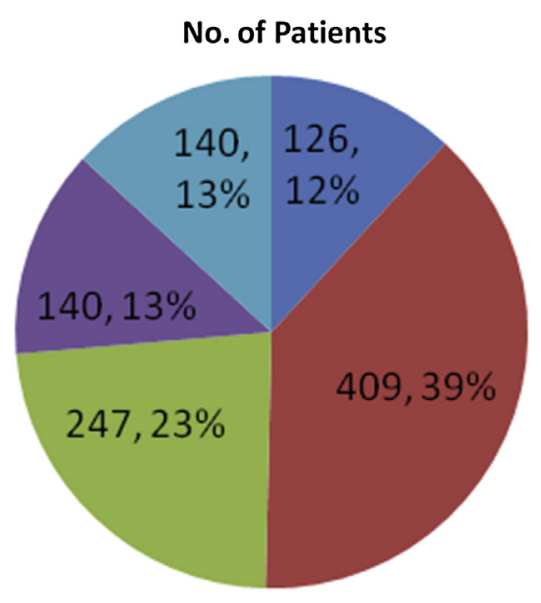

$A \square B \square C \square D \square$ Not Known

Figure 3 BCLC staging of HCC (AllMS 1990-2012). The figure depicts the BCLC staging and indicates that more than $50 \%$ of $\mathrm{HCC}$ patients of presentation has BCLC-A and B staging which were offered treatment where as a equal proportion of patient had advanced stage (BCLC-C and D). 


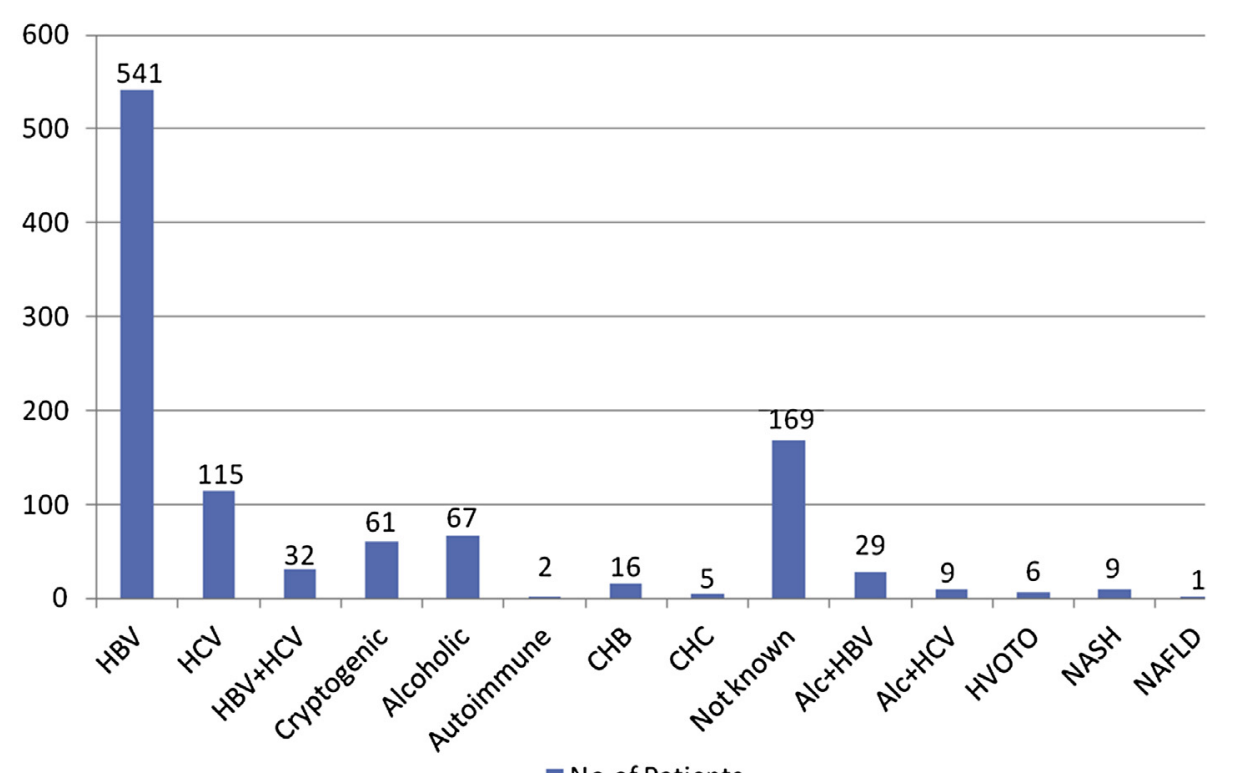

Figure 4 Etiology of HCC (AlIMS 1990-2012). The figure indicates the etiology of HCC in these 1062 patients which confirmed that HBV is the major cause of HCC in India as reported by other tertiary care centers from India. ${ }^{9,15,18,19}$

included total 952 cases. $^{9,15,18,19}$ However, none of these case series have provided HCC burden data in details, nor has provided the year wise breakup to assess whether HCC burden is increasing in the tertiary care centers. In absence of collated data from multiple tertiary care center, we evaluated our own center data (unpublished). From 1990 to 2012, 1062 consecutive patients with confirmed diagnosis were registered at the liver clinic at the All India Institute of Medical Sciences, New Delhi. Figure 1 provides the number of HCC presented every year which indicate that, there is progressive risk of number of HCC cases at our center. From 2006 we have been registering about 100 HCC patients per year (see Figure 2-4). HCC cases at our center. Figure 2-4 depicts the age distribution, BCLC staging and etiology of the HCC respectively in these cases at our center. These data indicates that $55 \%$ of the patients with HCC presented at late age (>62 years). About half of the patients had BCLC stage A and B in whom definitive therapy could be offered and 65\% (688/ 1062) of HCC were due to HBV and HCV.

We had access to some information from Christian Medical College (CMC) Vellore, Tamil Nadu and Sir Ganga Ram Hospital (SRGH) Delhi. At CMC, Vellore, between December 2003 and 2010, 331 cases of HCC were registered, indicating that approximately 60 patients with HCC are registered annually at this center. Forty of these patients were offered therapy.

At SRGH, Delhi between January 2000 and December 2012,142 patients with HCC were registered. The BCLC staging in these patients indicate that $13 \%$ of the patients had BCLC-A, $23 \%$ had BCLC-B, 35\% had BCLC-C and about $29 \%$ had BCLC-D. Eighty of these 142 patients were offered treatment.
Even though the information from tertiary center on HCC is limited, it seems that every tertiary care center registers about 50-60 HCC patients in a year. Approximately 320 tertiary care centers in India than would be registering approximately 16000 to 20,000 HCC cases in a year.

\section{REGIONAL DIFFERENCES ON HEPATOCELLULAR CARCINOMA BURDEN IN INDIA}

Clinicians practicing in country feels that the HCC burden in South India may be higher than remaining part of the country. The cancer registries indicate that the incidence of HCC in population at large is highest in north-eastern states. However, in absence of properly designed prospective study, comparing the burden of HCC in different region of the country, it may be difficult to assess the differences of regional burden in India.

\section{CONCLUSIONS}

Thus to conclude, the available data indicates that the age adjusted incidence rate of HCC in India for men ranges from 0.7 to 7.5 and for women 0.2 to 2.2 per 100,000 population per year. The incidence of HCC in cirrhotics in India is $1.6 \%$ per year. The male:female ratio for HCC in India is 4:1. The age of presentation varies from 40 to 70 years. The age standardized mortality rate for HCC in India for men is 6.8 / 100,000 and for women is $5.1 / 100,000$. The unpublished data from various tertiary care centers suggest that the incidence of HCC is increasing in India. There is a need for a multi-centric HCC registry under the aegis of INASL. 


\section{CONFLICTS OF INTEREST}

The author has none to declare.

\section{REFERENCES}

1. EASL-EORTC Clinical Practice Guideline. Management of hepatocellular carcinoma. J Hepatol. 2012;56:908-943.

2. Prabhakar V, Rao KS, Reddy DJ. Primary cancer of liver in Vishakhapatnam. Indian J Pathol Microbiol. 1966;9:54-60.

3. Dhir V, Mohandas KM. Epidemiology of digestive tract cancers in India III liver. Indian J Gastroenterol. 1998;17:100-103.

4. National Cancer Registry Program, ICMR http://ncrpindia.org/.

5. International Agency for Research on Cancer (IARC-WHO) http:// Ci5.iarc.fr (Access number 9).

6. Yuen F, Hou JC, Chuttapati A. Hepatocellular cancer in Asia Pacific region. J Gastroenterol Hepatol. 2009;24:346-353.

7. Yeole B. Trends in cancer incidences in esophagus, stomach, coIon, rectum and liver in males in India. Asian Pac $J$ Cancer Prev. 2008;9:97-100.

8. Dikshit R, Gupta PC, Ramasundarahelige C, et al, Million Death Study Collaborators. Lancet. 2012;79:1807-1816.

9. Mohandas KM. Surveillance of Indian with liver cirrhosis for treatable hepatocellular carcinoma: another enigma. J Gastroenterol. 2007;26:261-264.

10. Sarin SK, Thakur V, Guptan RC, et al. Hepatocellular cancer in India. J Gastroenterol Hepatol. 2001;16:666-673.

11. Paul SB, Chalamalasetty B, Vishnubatla S, et al. Clinical profile, etiology and therapeutic outcome in 324 hepatocellular cancer in India. Oncology. 2009;77:162-171.
12. Coon JT, Rogers G, Hewson P, et al. Surveillance of cirrhosis for hepatocellular carcinoma: systematic review and economic analysis. Health Technol Assess. 2007;11:1-206.

13. Fattovitch G, Stroffolini T, Zagni I, Donato F. Hepatocellular carcinoma in cirrhosis: incidence and risk factor (review). Gastroenterology. 2004;127(suppl I):S35-S50.

14. Chu CM. Natural history of chronic hepatitis B virus infection in adults with emphasis on the occurrence of cirrhosis and hepatocellular carcinoma. J Gastroenterol Hepatol. 2000;15(Sujppl-1): E25-E30.

15. Tanaka K, Sakai H, Hashizume M, Hirohata T. A long term follow up study on risk factors for hepatocellular carcinoma among Japanese patients with liver cirrhosis. Jpn J Cancer Res. 1998;89: 1241-1250.

16. Paul SB, Sreenivas V, Gulati MS, et al. Incidence of hepatocellular carcinoma among Indian patients with cirrhosis of liver: an experience from tertiary care center in northern India. Indian J Gastroenterol. 2007;26:274-278.

17. Battam A, Narula D, Tofeja T, Sreenivas V, Puliyel JM. Systematic review and meta analysis of prevalence of hepatitis $B$ in India. Indian Paediatr. 2007;44:663-674.

18. Ashim M, Sarma MP, Kar P. Etiological and molecular profile of hepatocellular cancer in India. Int J Cancer. 2013;133:437-445.

19. Sharma S, Sharma B, Chawla YK, et al. Comparison of 7 staging system in north Indian cohort of hepatocellular carcinoma. Trop Gastroenterol. 2010;35:271-278. 